\title{
PROBABILITÉS DE RUINE POUR UNE CLASSE DE MODÈLES \\ DE RISQUE SEMI-MARKOVIENS
}

\author{
By JaCQues JansSEN
}

Université Libre de Bruxelles

AND

JeAN-MARie Reinhard

Groupe $A G$

\begin{abstract}
We consider particular semi-Markov risk models $M / S M$ and $\bar{M} / S M$ for which the interarrival distributions are exponential with parameters depending of the risk type. We obtain theoretical expressions for the ruin probabilities on an infinite horizon.

In the special case of exponential distributions for the claim amounts, ruin probabilities are in both models solutions of linear differential systems. These systems are explicitly solved when there are only two risk types.
\end{abstract}

\section{MODÈLES CONSIDÉRÉS}

En suivant la terminologie de JANSSEN (1982), nous considérons essentiellement les modèles $M / S M$ et $\bar{M} / S M$ eux-mêmes cas particulier du modèle $S M / S M$ défini comme suit: $I=\{1, \ldots, m\}$ représentant l'ensemble des $m$ types de sinistres possibles, l'idée de base est de supposer que si $\left(J_{n}\right)_{n \geqslant 1},\left(Y_{n}\right)_{n \geqslant 1},\left(X_{n}\right)_{n \geqslant 1}$ représentent respectivement la suite des types successifs, la suite des montants successifs des sinistres et celle des interarrivées, nous supposons que

$$
\mathbb{P}\left[J_{n}=j, X_{n} \leqslant x, Y_{n} \leqslant y \mid\left(J_{k}, X_{k}, Y_{k}\right), k \leqslant n-1\right]=Q_{J_{n-1} j}(x, y)
$$

en admettant que $J_{0}=j_{0}, X_{0}=Y_{0}=0, j_{0} \in I$.

La matrice $Q(\cdot, \cdot)=\left(Q_{i j}(\cdot, \cdot)\right)$ est une matrice de fonctions de masse nulles en dehors de $\mathbb{R}^{+} \times \mathbb{R}^{+}$et telle que la matrice $P=Q(+\infty,+\infty)$ est une matrice de probabilité de transition, en fait celle de la chaîne de Markov $\left(J_{n}\right)$.

Le modèle $M / S M$ correspond au cas où:

$$
Q_{i j}(x, y)=p_{i j}^{A} F_{i}(x){ }^{B} F_{j}(y)
$$

avec

$$
{ }^{A} F_{i}(x)= \begin{cases}0, & x<0, \\ 1-e^{-\lambda_{i} x}, & x \geqslant 0 .\end{cases}
$$

Dans ces conditions, le processus des arrivées de sinistres est un processus de Markov homogène continu dans le temps ayant $I$ comme espace d'états. Le 
modèle $M^{\prime} / S M$ est une variante du précédent pour lequel

$$
Q_{i j}(x, y)=p_{i j}{ }^{A} F_{j}(x){ }^{B} F_{j}(y)
$$

où

$$
{ }^{A} F_{j}(x)= \begin{cases}0, & x<0 \\ 1-e^{-\lambda_{j} x}, & x \geqslant 0\end{cases}
$$

Il semble plus adéquat en effet de faire dépendre la distribution de l'interarrivée entre 2 sinistres successifs plutôt du sinistre qui suit que de celui qui précède.

Désignons par

$$
\begin{aligned}
& a_{j}=\int_{0}^{\infty} x^{A} d F_{j}(x), \\
& b_{j}=\int_{0}^{\infty} y^{B} d F_{j}(y),
\end{aligned}
$$

ces moyennes conditionnelles étant supposées finies. De plus, nous ferons toujours l'hypothèse que le taux de prime par unité de temps vaut 1 et ceci sans perte de généralité étant donné l'arbitraire laissé sur les moyennes $a_{j}$ et $b_{j}, j \in I$.

Enfin, la matrice $P$ sera toujours supposée ergodique ce qui implique l'existence d'une distribution stationnaire unique $\Pi=\left(\Pi_{1}, \ldots, \Pi_{m}\right)$.

Désignons, pour tout $i \in I$, par $R_{i}(u)$ la probabilité de non-ruine, partant en $t=0$, juste après l'arrivée du sinistre du type $i$ ayant, après règlement de ce sinistre, une réserve initiale de montant $u(u>0)$.

Il est bien connu que

$$
\lim _{u \rightarrow \infty} R_{i}(u)=1
$$

ssi

$$
\sum \Pi_{i}^{B} \eta_{i}<\sum \Pi_{i}{ }^{A} \eta_{i}
$$

où pour le modèle $M / S M$

$$
{ }^{B} \eta_{i}=\sum_{j} p_{i j} b_{j}, \quad{ }^{A} \eta_{i}=a_{i}
$$

et pour le modèle $M^{\prime} / S M$

$$
{ }^{B} \eta_{i}=\sum_{j} p_{i j} b_{j}, \quad{ }^{A} \eta_{i}=\sum_{j} p_{i j} a_{j} .
$$

Nous travaillons désormais en supposant la condition (1.9) remplie. Pour les deux modèles, on voit aisément que (1.9) est équivalent à:

$$
\sum_{j} \Pi_{j} b_{j}<\sum_{j} \Pi_{j} a_{j}
$$


2. TRAITEMENT DU MODÈlE $M / S M$ (JANSSEN, 1982)

Pour simplifier les notations, nous posons

$$
{ }^{B} F_{j}(\cdot)=B_{j}(\cdot) \text {. }
$$

Un raisonnement probabiliste élémentaire conduit au résultat suivant:

$$
R_{i}(u)=\sum_{j=1}^{m} p_{i j} \int_{0}^{\infty} \lambda_{i} e^{-\lambda_{i} \tau} d \tau \int_{0}^{u+\tau} R_{j}(u+\tau-x) d B_{j}(x), \quad i \in I .
$$

En effectuant le changement de variable $u+\tau=\xi$, il vient:

$$
R_{i}(u)=\sum_{j=1}^{m} \lambda_{i} p_{i j} \int_{u}^{\infty} e^{-\lambda_{i}(\xi-u)} d \xi \int_{0}^{\xi} R_{j}(\xi-x) d B_{j}(x)
$$

ce qui prouve la différentiabilité des $R_{i}(u)$. La différentiation donne:

$$
R_{i}^{\prime}(u)=\lambda_{i} R_{i}(u)-\lambda_{i} \sum_{j=1}^{m} p_{i j} \int_{0}^{u} R_{j}(u-x) d B_{j}(x), \quad i \in I .
$$

De façon générale, en désignant par $\tilde{C}$ la transformée de Laplace de la fonction $C$ :

$$
\tilde{C}(s)=\int_{0}^{\infty} e^{-s t} C(t) d t
$$

le système précédent prend la forme:

$$
s \tilde{R}_{i}(s)+\lambda_{i} \sum_{j=1}^{m}\left[p_{i j} b_{j}(s)-\delta_{i j}\right] \tilde{R}_{j}(s)=R_{i}(0)
$$

où

$$
b_{j}(s)=\int_{0^{-}}^{\infty} e^{-s x} d B_{j}(x)
$$

Posons maintenant:

$$
\begin{gathered}
\Lambda=\left(\lambda_{i} \delta_{i j}\right), \\
b(s)=\left(\delta_{i j} b_{j}(s)\right) .
\end{gathered}
$$

Si $\tilde{R}(s)$ représente le vecteur-colonne des $\tilde{R}_{i}(s), i=1, \ldots, m$ et $R(u)$ celui des $R_{i}(u)$, le système $(2.6)$ devient:

$$
s\left(I-\Lambda\left(\frac{I-P b(s)}{s}\right)\right) \tilde{R}(s)=R(0) .
$$

On vérifie aisément que la matrice

$$
\tilde{A}(s)=\frac{\Lambda(I-P b(s))}{s}
$$

tend vers la matrice nulle lorsque $s$ tend vers $\infty$; par conséquent. 


$$
(I-\tilde{A}(s))^{-1}=\sum_{n=0}^{\infty}(\tilde{A}(s))^{n}
$$

Posons

$$
A(t)=\mathscr{L}^{-1}(\tilde{A}(s))
$$

Il vient

$$
A(t)=\Lambda(I-P B(t))
$$

où $B(\cdot)$ est la matrice diagonale dont les éléments diagonaux sont $B_{j}(\cdot)$. Nous avons ainsi la forme explicite de la transformée de Laplace inverse de $(I-\tilde{A}(s))^{-1}$ par

$$
\mathscr{L}^{-1}(I-\tilde{A}(s))^{-1}=\sum_{n=0}^{\infty} A^{(n)}(t)
$$

$A^{(n)}(\cdot)$ représentant la $n^{i e ̀ m e}$ convoluée de la matrice $A(\cdot)$ :

$$
\begin{gathered}
A^{(0)}(t)=I(t), \quad A^{(1)}(t)=A(t), \\
A^{(n)}(t)=\left(\sum_{j=1}^{m} \int_{0}^{t} A_{i j}^{(n-1)}(t-v) A_{j k}(v) d v\right), \quad n \geqslant 2 .
\end{gathered}
$$

En revenant au système $(2.9)$, il vient:

$$
R(u)=\left(\sum_{n=0}^{\infty} \int_{0}^{u} A^{(n)}(t) d t\right) R(0)
$$

la valeur de $R(0)$ résultant du fait que, par (1.8):

$$
\lim _{u \rightarrow \infty} R(u)=1 \text {. }
$$

Si $m=1$, on retrouve le résultat suivant:

$$
R(u)=\left(1+\sum_{n=1}^{\infty} \lambda^{n} \int_{0}^{u} \bar{B}^{(n)}(t) d t\right) R(0)
$$

où

$$
\bar{B}(u)=1-B(u) .
$$

En faisant tendre $u \rightarrow \infty$, il vient:

$$
\begin{aligned}
R(0) & =\left(1+\sum_{n=1}^{\infty} \lambda^{n} b^{n}\right)^{-1} \\
& =1-\lambda b
\end{aligned}
$$

$b$ étant la moyenne relative à la f.d. $B(\cdot)$. 


\section{TRAITEMENT DU MODÈLE $M^{\prime} / S M$}

Montrons d'abord que pour un tel modèle le processus

$$
\left(\left(J_{n+1}, X_{n}, Y_{n}\right), n \geqslant 0\right)
$$

vérifie encore (1.1); en effet, on a:

$$
\begin{aligned}
\mathbb{P}\left[J_{n+1}\right. & \left.=l, X_{n} \leqslant x, Y_{n} \leqslant y \mid\left(J_{l+1}, X_{l}, Y_{l}\right), l \leqslant n-1, J_{n}=k\right] \\
& =p_{k l}{ }^{A} F_{k}(x){ }^{B} F_{k}(y)
\end{aligned}
$$

Désignons par $\bar{R}_{i}(u)$ les probabilités de non-ruine relatives au noyau (3.1). Il est clair que

$$
R_{i}(u)=\sum_{j} p_{i j} \bar{R}_{j}(u)
$$

Il suffit donc de calculer les probabilités $\bar{R}_{j}(\cdot)$ pour en déduire les probabilités $R_{i}(u)$ cherchées.

Un raisonnement analogue à celui fait à la Section 2 donne:

$$
\bar{R}_{i}(u)=\sum_{j} p_{i j} \int_{0}^{\infty} \lambda_{i} e^{-\lambda_{i} \tau} d \tau \int_{0}^{u+\tau} \bar{R}_{j}(u+\tau-x) d B_{i}(x)
$$

En effectuant le changement de variables $u+\tau=\xi$, il vient

$$
\bar{R}_{i}(u)=\sum_{j} \lambda_{i} p_{i j} \int_{0}^{\infty} e^{-\lambda_{i}(\xi-u)} d \xi \int_{0}^{\xi} \bar{R}_{j}(\xi-x) d B_{i}(x)
$$

ce qui prouve la différentiabilité des $R_{i}(u)$. La différentiation donne:

$$
\bar{R}_{i}^{\prime}(u)=\lambda_{i} \bar{R}_{i}(u)-\lambda_{i} \sum_{j} p_{i j} \int_{0}^{u} \bar{R}_{j}(\xi-x) d B_{i}(x)
$$

systéme analogue au système (2.4) et qui se traite de la même façon. En passant aux transformées de Laplace, on obtient:

$$
s \tilde{\bar{R}}_{i}(s)+\lambda_{i} \sum_{j=1}^{m}\left[p_{i j} b_{i}(s)-\delta_{i j}\right] \tilde{\bar{R}}_{j}(s)=\bar{R}_{i}(0)
$$

Posons maintenant

$$
\begin{gathered}
\Lambda=\left(\lambda_{i} \delta_{i j}\right), \\
b(s)=\left(\delta_{i j} b_{j}(s)\right) .
\end{gathered}
$$

Le système (3.6) devient

$$
s\left(I-\frac{\Lambda(I-b P)}{s}\right) \tilde{R}=\bar{R}(0),
$$

expression analogue à (2.9). 
Posons

$$
\tilde{A}(s)=\frac{\Lambda(I-b P)}{s}
$$

tendant à nouveau vers la matrice nulle lorsque $s$ tend vers $+\infty$; par conséquent:

$$
(I-\tilde{\tilde{A}}(s))^{-1}=\sum_{n=0}^{\infty}(\underline{\tilde{A}}(s))^{n} .
$$

Posons

$$
\underline{A}(t)=\mathscr{L}^{-1}(\underline{\tilde{A}}(s)) .
$$

Il vient

$$
\underline{A}(t)=\Lambda(I-B(t) P),
$$

où $B(\cdot)$ est la matrice diagonale dont les éléments diagonaux sont $B_{j}(\cdot)$. Il vient ainsi:

$$
\mathscr{L}^{-1}(I-\tilde{A}(s))^{-1}=\sum_{n=0}^{\infty} \underline{A}^{(n)}(t)
$$

les convoluées successives de $\underline{A}(t)$ étant définies par des relations identiques à (2.15) et (2.16).

En revenant au système, il vient:

$$
\bar{R}(u)=\left(\sum_{n=0}^{\infty} \int_{0}^{u} \underline{A}^{(n)}(u) d t\right) \bar{R}(0)
$$

$\bar{R}(0)$ étant définie par la relation (2.18).

Evidemment, le cas particulier $m=1$ redonne (2.19) puisque dans ce cas, les matrices $B(\cdot)$ et $P$ commutent.

Cas particulier:

$$
b_{j}(x)=\mu_{j} e^{-\mu_{j} x} \quad \text { où } \quad \mu_{j}=b_{j}^{-1}, \quad j=1, \ldots, m .
$$

Dans ce cas, les relations (3.5) deviennent:

$$
\bar{R}_{i}^{\prime}(u)=\lambda_{i} \bar{R}_{i}(u)-\lambda_{i} \sum_{j} p_{i j} \int_{0}^{u} \bar{R}_{j}(x) \mu_{i} e^{-\mu_{i}(u-x)} d x
$$

d'où l'existence des dérivées secondes des fonctions $R_{i}(\cdot)$ qui se mettent sous la forme:

$$
\begin{aligned}
\bar{R}_{i}^{\prime \prime}(u) & =\lambda_{i} \bar{R}_{i}^{\prime}(u)-\lambda_{i} \sum_{j} p_{i j}\left[-\int_{0}^{u} \mu_{i}^{2} \bar{R}_{j}(x) e^{-\mu_{i}(u-x)} d x+\bar{R}_{j}(u) \mu_{i}\right] \\
& =\lambda_{i} \bar{R}_{i}^{\prime}(u)-\mu_{i}\left[\lambda_{i} \bar{R}_{i}(u)-\bar{R}_{i}^{\prime}(u)\right]-\lambda_{i} \mu_{i} \sum_{j} P_{i j} \bar{R}_{j}(u)
\end{aligned}
$$


On voit ainsi que les fonctions $\bar{R}_{1}(\cdot), \ldots, \bar{R}_{m}(\cdot)$ satisfont le système différentiel du second ordre à coefficients constants:

$$
\bar{R}_{i}^{\prime \prime}(u)=\left(\lambda_{i}-\mu_{i}\right) \bar{R}_{i}^{\prime}(u)+\lambda_{i} \mu_{i} \bar{R}_{i}(u)-\lambda_{i} \mu_{i} \sum_{j} p_{i j} \bar{R}_{j}(u)
$$

Si $m=1$, on retrouve l'équation

$$
R^{\prime \prime}(u)=:(\lambda-u) R^{\prime}(u)
$$

discutée dans Gerber (1979).

L'intérêt de (3.20) est de mettre la solution générale sous la forme d'une combinaison linéaire en général à coefficients constants de $2 m$ exponentielles négatives ce qui est d'un grand intérêt, comme les développements ci-dessous le montrent, pour l'obtention de résultats numériques.

REMARQUE. (3.3) se met encore, grâce à (3.2) sous la forme:

$$
\bar{R}_{i}(u)=\lambda_{i} \int_{0}^{\infty} e^{-\lambda_{i} \tau} d \tau \int_{0}^{u+\tau} R_{i}(u+\tau-x) d B_{i}(x)
$$

relations "inverses" de (3.2).

\section{LE CAS EXPONENTIEL}

Nous montrons que lorsque les distributions des montants de sinistres sont exponentielles:

$$
B_{j}(x)=1-e^{-x / b_{j}}, \quad(x \geqslant 0, j \in I)
$$

le calcul des probabilités de non-ruine pour chacun des deux modèles traités ci-dessus se ramène à la résolution d'un système différentiel linéaire du deuxième ordre. Nous donnons explicitement la solution de ces systèmes dans le cas particulier $m=2$. Nous supposerons constamment la condition (1.12) satisfaite.

\subsection{Le Modèle $M / S M$}

4.1.1. Sous la condition (4.1), on peut réécrire (2.4) comme suit:

$$
R_{i}^{\prime}(u)=\lambda_{i} R_{i}(u)=\lambda_{i} \sum_{j} p_{i j} \frac{1}{b_{j}} \int_{0}^{u} R_{j}(y) e^{-(u-y) / b_{j}} d y
$$

d'où l'on déduit par une nouvelle dérivation

$$
\begin{aligned}
R_{i}^{\prime \prime}(u)= & \lambda_{i} R_{i}^{\prime}(u)-\lambda_{i} \sum_{j} p_{i j} \frac{1}{b_{j}} R_{j}(u) \\
& +\lambda_{i} \sum_{j} p_{i j} \frac{1}{b_{j}^{2}} \int_{0}^{u} R_{j}(y) e^{-(u-y) / b_{j}} d y .
\end{aligned}
$$

On ne peut ramener directement ce systéme d'équations intégro-différentielles à un système différentiel. Cependant, on peut, pour se ramener à un système 
différentiel utiliser l'artifice suivant. Soit $L_{j}(u)$ la probabilité de non-ruine asymptotique juste avant l'arrivée d'un sinistre de type $j$ si le montant des réserves libres avant ce sinistre est $u$. Ces probabilités sont liées aux $R_{i}(u)$ par les relations

$$
R_{i}(u)=\sum_{j} p_{i j} \int_{0}^{\infty} \lambda_{i} e^{-\lambda_{i} t} L_{j}(u+t) d t, \quad(u \geqslant 0, i \in I) .
$$

Un raisonnement probabiliste classique montre par ailleurs que les probabilités $L_{j}(u)$ vérifient les relations

$$
\begin{aligned}
L_{j}(u)= & \int_{0}^{u} \frac{1}{b_{j}} e^{-x / b_{j}} \int_{0}^{\infty} \lambda_{j} e^{-\lambda_{j} t} \\
& \times \sum_{k=1}^{m} p_{j k} L_{k}(u+t-x) d t d x, \quad(j \in I, u \geqslant 0)
\end{aligned}
$$

ou encore en posant $u-x=z$ :

$$
\begin{aligned}
L_{j}(u)= & \int_{0}^{u} \frac{1}{b_{j}} e^{-(u-z) / b_{j}} \int_{0}^{\infty} \lambda_{j} e^{-\lambda_{j} t} \\
& \times \sum_{k=1}^{m} p_{j k} L_{k}(z+t) d t d z, \quad(j \in I, u \geqslant 0) .
\end{aligned}
$$

Dérivons les deux membres de (4.6) par rapport à $u$ et posons ensuite $u+t=v$. Il vient:

$$
L_{j}^{\prime}(u)=\frac{\lambda_{j}}{b_{j}} \int_{u}^{\infty} e^{-\lambda_{j}(v-u)} \sum_{k} p_{j k} L_{k}(v) d v-\frac{1}{b_{j}} L_{j}(u), \quad(j \in I, u \geqslant 0) .
$$

Dérivons une nouvelle fois les deux membres de (4.7) par rapport à $u$; on obtient

$$
\begin{aligned}
L_{j}^{\prime \prime}(u)= & -\frac{\lambda_{j}}{b_{j}} \sum_{k} p_{j k} L_{k}(u)+\lambda_{j}^{2} \frac{1}{b_{j}} \int_{u}^{\infty} e^{-\lambda_{j}(v-u)} \\
& \times \sum_{k} p_{j k} L_{k}(v) d v-\frac{1}{b_{j}} L_{j}^{\prime}(u), \quad(j \in I, u \geqslant 0)
\end{aligned}
$$

ou encore en réutilisant (4.7):

$$
L_{j}^{\prime \prime}(u)+\left(\frac{1}{b_{j}}-\lambda_{j}\right) L_{j}^{\prime}(u)-\frac{\lambda_{j}}{b_{j}}\left[L_{j}(u)-\sum_{k} p_{j k} L_{k}(u)\right]=0 .
$$

Le système différentiel linéaire (4.9) doit être résolu avec les conditions aux bornes

$$
L_{j}(0)=0, \quad L_{j}(\infty)=1, \quad(j \in I)
$$

la deuxième de ces conditions résultant de (1.12) et (4.4).

4.1.2. Considérons le cas particulier où $m=2$ et où $p_{12} \cdot p_{21}>0$ (cette dernière condition résultant d'ailleurs du fait que la matrice $P$ est supposée ergodique). 
Le système (4.9) présente alors la même structure que le système rencontré dans ReINHARD (1984):

$$
\left\{\begin{array}{l}
L_{1}^{\prime \prime}(u)+\left(\frac{1}{b_{1}}-\lambda_{1}\right) L_{1}^{\prime}(u)-\frac{\lambda_{1} p_{12}}{b_{1}} L_{1}(u)+\frac{\lambda_{1} p_{12}}{b_{1}} L_{2}(u)=0 \\
L_{2}^{\prime \prime}(u)+\left(\frac{1}{b_{2}}-\lambda_{2}\right) L_{2}^{\prime}(u)-\frac{\lambda_{2} p_{21}}{b_{2}} L_{2}(u)+\frac{\lambda_{2} p_{21}}{b_{1}} L_{1}(u)=0
\end{array}\right.
$$

Posons

$$
\rho_{i}=\left(\frac{1}{b_{i}}-\lambda_{i}\right), \quad(i=1,2)
$$

et supposons sans restriction $\rho_{1} \geqslant \rho_{2}$; on a alors, vu (1.12), $\rho_{1}>0$.

La solution générale de (4.11) est donnée par

$$
\left\{\begin{array}{l}
L_{1}(u)=A_{0}+A_{1} e^{k_{1} u}+A_{2} e^{k_{2} u}+A_{3} e^{k_{3} u} \\
L_{2}(u)=A_{0}-D\left(k_{1}\right) A_{1} e^{k_{1} u}-D\left(k_{2}\right) A_{2} e^{k_{2} u}-D\left(k_{3}\right) A_{3} e^{k_{3} u}
\end{array}\right.
$$

où $k_{1}, k_{2}$ et $k_{3}$ sont les racines de l'équation du 3ème degré

$$
\begin{aligned}
P(k)= & k^{3}+\left(\rho_{1}+\rho_{2}\right) k^{2}+\left(\rho_{1} \rho_{2}-\frac{\lambda_{2}}{b_{2}} p_{21}-\frac{\lambda_{1}}{b_{1}} p_{12}\right) k \\
& -\left(\frac{\lambda_{2} \rho_{1}}{b_{2}} p_{21}+\frac{\lambda_{1} \rho_{2}}{b_{1}} p_{12}\right)=0
\end{aligned}
$$

et où

$$
D\left(k_{i}\right)=\frac{b_{1}\left(k_{i}^{2}+\rho_{1} k_{i}\right)}{\lambda_{1} p_{12}}-1, \quad(i=1,2,3)
$$

Comme

$$
\Pi_{1}=\frac{p_{21}}{p_{12}+p_{21}}, \quad \Pi_{2}=\frac{p_{12}}{p_{12}+p_{21}}
$$

(1.12) est équivalent à

$$
\frac{\lambda_{2} \rho_{1}}{b_{2}} p_{21}+\frac{\lambda_{1} \rho_{2}}{b_{1}} p_{12}>0
$$

Par conséquent $k_{1} k_{2} k_{3}>0$. Comme

$$
\left\{\begin{array}{l}
P(0)<0, \quad P(+\infty)=+\infty, \quad P(-\infty)=-\infty, \\
p\left(-\rho_{1}\right)=\frac{\lambda_{1}}{b_{1}} p_{12}\left(\rho_{1}-\rho_{2}\right) \geqslant 0, \\
P\left(-\rho_{2}\right)=\frac{\lambda_{2}}{b_{2}} p_{21}\left(\rho_{2}-\rho_{1}\right) \leqslant 0,
\end{array}\right.
$$


$P$ a une racine négative, soit $k_{2}$ satisfaisant

$$
-\rho_{1} \leqslant k_{2} \leqslant-\rho_{2}
$$

èt une racine positive, soit $k_{3}$. On voit finalement que si $\rho_{1}>\rho_{2}$, la troisième racine $k_{1}$ est strictement inférieure à $-\rho_{1}$. Si $\rho_{1}=\rho_{2}=\rho$ on obtient la même conclusion en vérifiant que $P^{\prime}(-\rho)<0$. En résumé:

$$
\begin{cases}k_{1}<-\rho_{1}<k_{2}<\min \left\{0,-\rho_{2}\right\} ; k_{3}>0 & \text { si } \rho_{1}>\rho_{2}, \\ k_{1}<-\rho=k_{2}<0<k_{3} & \text { si } \rho_{1}=\rho_{2}=\rho .\end{cases}
$$

La condition $L_{1}(\infty)=L_{2}(\infty)=1$ entraîne alors $A_{3}=0, A_{0}=1$. $A_{1}$ et $A_{2}$ sont alors déterminés par la condition $L_{1}(0)=L_{2}(0)=0$ :

$$
\left\{\begin{array}{l}
A_{1}+A_{2}=-1 \\
-D\left(k_{1}\right) A_{1}-D\left(k_{2}\right) A_{2}=-1
\end{array}\right.
$$

d'où

$$
A_{1}=\frac{1+D\left(k_{2}\right)}{D\left(k_{1}\right)-D\left(k_{2}\right)}, \quad A_{2}=\frac{1+D\left(k_{1}\right)}{D\left(k_{2}\right)-D\left(k_{1}\right)}
$$

Finalement:

$$
\left\{\begin{array}{l}
L_{1}(u)=1+\frac{1+D\left(k_{2}\right)}{D\left(k_{1}\right)-D\left(k_{2}\right)} e^{k_{1} u}+\frac{1+D\left(k_{1}\right)}{D\left(k_{2}\right)-D\left(k_{1}\right)} e^{k_{2} u} \\
L_{2}(u)=1-D\left(k_{1}\right) \frac{1+D\left(k_{2}\right)}{D\left(k_{1}\right)-D\left(k_{2}\right)} e^{k_{1} u}-D\left(k_{2}\right) \frac{1+D\left(k_{1}\right)}{D\left(k_{2}\right)-D\left(k_{1}\right)} e^{k_{2} u}
\end{array}\right.
$$

Les probabilités de non ruine $R_{i}(u)$ peuvent alors être explicitées en utilisant (4.4) et (4.22). On obtient:

$$
\left\{\begin{aligned}
R_{1}(u)= & 1+\frac{\lambda_{1}}{\lambda_{1}-k_{1}}\left(p_{11}-p_{12} D\left(k_{1}\right)\right) A_{1} e^{k_{1} u} \\
& +\frac{\lambda_{1}}{\lambda_{1}-k_{2}}\left(p_{11}-p_{12} D\left(k_{2}\right)\right) A_{2} e^{k_{2} u} \\
R_{2}(u)= & 1+\frac{\lambda_{2}}{\lambda_{2}-k_{1}}\left(p_{21}-p_{22} D\left(k_{1}\right)\right) A_{1} e^{k_{1} u} \\
& +\frac{\lambda_{2}}{\lambda_{2}-k_{2}}\left(p_{21}-p_{22} D\left(k_{2}\right)\right) A_{2} e^{k_{2} u}
\end{aligned}\right.
$$

\subsection{Le Modèle $M^{\prime} / S M$}

4.2.1. Sous la condition (4.1) le système (3.5) s'écrit

$$
\bar{R}_{i}^{\prime}(u)=\lambda_{i} \bar{R}_{i}(u)-\frac{\lambda_{i}}{b_{i}} \sum_{j} p_{i j} \int_{0}^{u} \bar{R}_{j}(y) e^{-(u-y) / b_{i}} d y, \quad(i \in I, u \geqslant 0) .
$$


Dérivons une nouvelle fois les deux membres de (4.24). Il vient

$$
\bar{R}_{i}^{\prime \prime}(u)+\left(\frac{1}{b_{i}}-\lambda_{i}\right) \bar{R}_{i}^{\prime}(u)-\frac{\lambda_{i}}{b_{i}}\left[\bar{R}_{i}(u)-\sum_{k} p_{i k} \bar{R}_{k}(u)\right], \quad(i \in I, u \geqslant 0),
$$

c'est-à-dire un système différentiel identique à (4.9). Il faut cette fois le résoudre avec les conditions aux bornes

$$
\bar{R}_{i}(\infty)=1, \quad \bar{R}_{i}^{\prime}(0)=\lambda_{i} R_{i}(0), \quad(i \in I) .
$$

4.2.2. Dans le cas particulier $m=2, p_{12} \cdot p_{21}>0$, on obtient en conservant les notations de la Section 4.1.2:

$$
\left\{\begin{array}{l}
\bar{R}_{1}(u)=1+\bar{A}_{1} e^{k_{1} u}+\bar{A}_{2} e^{k_{2} u} \\
\bar{R}_{2}(u)=1-D\left(k_{1}\right) \bar{A}_{1} e^{k_{1} u}-D\left(k_{2}\right) \bar{A}_{2} e^{k_{2} u}
\end{array}\right.
$$

où $\overline{A_{1}}$ et $\overline{A_{2}}$ sont solutions de

$$
\left\{\begin{array}{l}
\left(k_{1}-\lambda_{1}\right) \bar{A}_{1}+\left(k_{2}-\lambda_{2}\right) \bar{A}_{2}=\lambda_{1}, \\
-\left(k_{1}-\lambda_{1}\right) D\left(k_{1}\right) \bar{A}_{1}-\left(k_{2}-\lambda_{2}\right) D\left(k_{2}\right) \bar{A}_{2}=\lambda_{2}
\end{array}\right.
$$

Les probabilités de non-ruine $R_{i}(u)$ du modèle initial sont alors déterminées par (4.27) et (3.2). On obtient après calculs:

$$
\left\{\begin{array}{l}
R_{1}(u)=1+\left[1-\frac{k_{1}\left(k_{1}+\rho_{1}\right)}{\lambda_{1} b_{1}}\right] \bar{A}_{1} e^{k_{1} u}+\left[1-\frac{k_{2}\left(k_{2}+\rho_{1}\right)}{\lambda_{1} b_{1}}\right] \bar{A}_{2} e^{k_{2} u} \\
R_{2}(u)=1+\left[1-\frac{p_{22} k_{1}\left(k_{1}+\rho_{1}\right)}{p_{12} \lambda_{1} b_{1}}\right] \bar{A}_{1} e^{k_{1} u}+\left[1-\frac{p_{22} k_{2}\left(k_{2}+\rho_{1}\right)}{p_{12} \lambda_{1} b_{1}}\right] \bar{A}_{2} e^{k_{2} u}
\end{array}\right.
$$

\section{RÉFÉRENCES}

Gerber, H. U. (1979) An Introduction to Mathematical Risk Theory. Richard D. Irwin, Inc.: Homewood.

JANSSEN, J. (1982) Modèles de risque semi-markoviens. Cahiers du C.E.R.O. 24, 261-280.

Reinhard, J. M. (1984) On a Class of Semi-Markov Risk Models Obtained as Classical Risk Models in a Markovian Environment. Astin Bulletin 14, 23-43. 